sub-groups to target with interventions, and propose topics for further research.

Method. A literature search was performed with Ovid on three databases, using wildcards and synonyms to increase the number of hits. This search produced 379 results, of which 41 remained after inclusion/exclusion criteria were applied. Additional sources were utilised as the review was written.

Result. Strong family relationships are protective against illicit substance use for under-25s, with conflicting results for licit substance use. Healthy peer relationships protect against substance use, particularly in the academically-stressful university environment. All Jamaican under-25s appear to be susceptible to peer pressure, which increases the likelihood of substance use. Spirituality is protective against substance use, although male Rastafarians are more likely to use cannabis. Certain forms of childhood maltreatment make use of particular substances more likely. University students and under-18s brought up in singleparent families are key sub-groups to target with interventions. Further research on mechanisms by which these determinants work, particular religions and which determinant has the greatest effect is recommended.

Conclusion. Various factors can protect against or predispose substance use in Jamaican under-25s. This review, and future research, can help inform policy decisions and intervention design for the key sub-groups found.

\section{Long admission waiting list at the Orchard clinic-why?}

Hannah Sayeed ${ }^{1 \star}$, Johanna Brown ${ }^{2}$ and Fionnbar Lenihan ${ }^{3}$

${ }^{1}$ ST6 Forensic Psychiatry, the Orchard clinic, NHS Lothian; ${ }^{2}$ Clinical director and Consultant Forensic psychiatrist, the Orchard Clinic, NHS Lothian and ${ }^{3}$ Consultant psychiatrist, St Bricin's Military Hospital ${ }^{*}$ Corresponding author.

doi: $10.1192 /$ bjo. 2021.763

Aims. The Orchard clinic is one of the three medium secure units in Scotland. This project was completed:

To gain an understanding of the causes of the Orchard clinic's long admission waiting list.

To use this information to improve current clinical pathways, service development and further research and development.

Method. To study the longitudinal traffic flow through the clinic from January 2017 to December 2019, data were collected for this time retrospectively from electronic minutes of fortnightly bed management meetings at the Orchard clinic.

This was cross checked with the Orchard clinic's record of admissions and discharges during this time and approved by the Forensic Research and Audit Group, NHS Lothian.

Result. November 2018 onwards, a surge of $90 \%$ was observed in the admission waiting list.

Looking at the trends of traffic flow through the clinic during this time, the following observations were made:

1. More admissions than discharges, especially November 2018 onwards.

2. New referrals for medium secure care at the Orchard clinic peaked twice during this time.

3. Delayed discharges peaked in July 2018 and further in January 2019 running parallel to the surge in admission waiting list thereafter.

4. $42 \%$ patients on the delayed discharge list belonged to other health boards awaiting local low secure/community placements.
Conclusion. Delayed discharges were identified as a constant parallel to the long waiting list and hence identified as the main factor contributing to it. Out of area (non-NHS Lothian) admissions were noted to be linked to these delayed discharges.

Regular peaks in new referrals was also noted to be contributory.

\section{A randomised controlled trial to investigate the} effectiveness of sustained photoprotective behaviour in xeroderma pigmentosum after intervention

Tamara Searle $^{1 \star}$, Jessica Walburn ${ }^{1}$ and Sam Norton ${ }^{2}$

${ }^{1}$ IoPNN King's College London, University of Birmingham and

${ }^{2}$ IoPPN King's College London

${ }^{\star}$ Corresponding author.

doi: 10.1192/bjo.2021.764

Aims. This study aimed to investigate whether an intervention designed to improve photoprotective behaviours is effective at changing behaviour and whether any change could be maintained.

Background. Xeroderma Pigmentosum (XP) is a rare condition in which patients are at risk of malignancies when exposed to ultra-violet radiation (UVR). Sufferers must take extra precautions to protect themselves from UVR. They must apply sunscreen to exposed skin, wear thick clothing, gloves, and a UVR-protective visor. Treatments include preventative photoprotective measures; the use of sunscreen and protective clothing. Additionally, frequent eye and skin examinations are required and swift removal of any premalignant lesions.

Method. In this randomised controlled trial, 16 participants with XP were given questionnaires at 4 time points; baseline, post-intervention, 5 months and 9 months post-intervention. The intervention involved 7 one-on-one counselling sessions, as well as telephone consultations. Counselling sessions encouraged photoprotection adherence, self-efficacy and discussions of any barriers to improving photoprotective behaviour. This study focused on psychosocial variables, attitudes and photoprotection. Questionnaires included the photoprotection self-efficacy questionnaire, Self-Reported Behavioural Automaticity Index, Short Warwick-Edinburgh Mental Wellbeing Scale, Quality of Life and Brief Photoprotection Adherence Questionnaire.

Result. The intervention was shown to have no significant effect on participants' questionnaires scores. Univariate ANCOVA revealed a group effect between follow-up 1 (FU1) and follow-up 2 (FU2); $\eta 2=0.422$ for self-efficacy in wearing photoprotective clothing. A group effect was identified from BL to FU1 and FU1 to FU2; $\eta 2=0.343$ and $\eta 2=0.378$ respectively in how often participants reapplied sunscreen to their face when outside for longer periods. Univariate ANCOVA revealed no group or time effect for the other outcome variables; for example, sunscreen self-efficacy.

Conclusion. The intervention had no significant effect on photoprotective behaviour questionnaire scores. Future research could focus on recruiting more participants globally to generate more statistically powered results. Research should focus on producing a maintainable intervention so that any positive change would produce better long-term health outcomes. This study lays the foundations for future XP research, which will be vital to improve understanding and enhance photo protective behaviour. 
How does self-report of anxiety symptoms compare with observer assessments after acquired brain injury?

Alex Seelochan $^{1 \star}$, Mark Paramlall ${ }^{2}$, Himanshu Tyagi ${ }^{3}$, Rohan Kandasamy ${ }^{4}$, Ida Bakar ${ }^{5}$, Cameron Holloway ${ }^{6}$, Samantha Harding ${ }^{7}$ and Anna Gadhvi ${ }^{2}$

${ }^{1}$ Northern Ontario School of Medicine; ${ }^{2}$ North Bristol NHS Trust, Frenchay Brain Injury Rehabilitation Centre; ${ }^{3}$ The National Hospital for Neurology and Neurosurgery, University College London; ${ }^{4}$ The National Hospital for Neurology and Neurosurgery, Frenchay Brain Injury Rehabilitation Centre; ${ }^{5}$ Frenchay Brain Injury Rehabilitation Centre, North Bristol NHS Trust; ${ }^{6}$ Frenchay Brain Injury Rehabilitation Centre and ${ }^{7}$ North Bristol NHS Trust ${ }^{\star}$ Corresponding author.

\section{doi: 10.1192/bjo.2021.765}

Aims. Comorbid anxiety and mood disorders occur in $30 \%$ and $60 \%$ of individuals post-ABI (acquired brain injury), respectively (Juengst et al, 2014). The presence of psychiatric symptoms correlate to poorer outcomes in post-stroke rehabilitation, worsened quality of life (QoL), and deficits in memory, attention, and processing speed that persists years following the index event. Despite this, it is unclear whether to what degree anxiety impacts cognition. Furthermore, the literature on this topic is inconsistent when comparing subjective and clinician measurements. This study seeks to ameliorate this gap in literature by analyzing how clinicians' measures of anxiety and cognitive performance correlate with subjective assessments of patient's own anxiety symptoms.

Method. Individuals with an ABI who were seen in a clinical neuropsychiatry outpatient clinic between 2019 and 2020 completed a GAD-7 (Generalized Anxiety Disorder-7) questionnaire (patient's self-report of the severity of anxiety symptoms) and an observer completed a Neuropsychiatric Inventory Questionnaire (NPIQ) including a subscale for anxiety (NPIQ-A). Participants also underwent a formal cognitive examination with the Montreal Cognitive Assessment (MoCA). A total of $24 \mathrm{ABI}$ patients (depressed ABI and nondepressed $\mathrm{ABI}$ ) were analyzed for variation, statistical agreement and correlation. Here, total anxiety scores (using GAD-7 scores), anxiety severity (correlating category based on total GAD-7 score) were compared against the objective measures for anxiety (NPI-QA) and cognition (MoCA). In order to standardize MoCA scores, $\mathrm{z}$ scores were used in the statistical analysis.

Result. The patient's subjective raw scores of anxiety were statistically significantly different from the corresponding scores from objective observers on Wilcoxon-Rank Sum tests $(p<0.01)$, however, there was a statistical correlation between GAD (categorized by severity level) and NPI-QA $(p=0.75)$. Spearman Rank Correlation did show positive, but, statistically insignificant correlation between dyads of these independent variables (including GAD7/NPIQ-A, GAD 7 categorised/NPIQ-A, GAD7/MoCA, GAD 7 categorised/MoCA).

Conclusion. These findings indicate (1) self-reported measures of anxiety (GAD7) in ABI were inconsistent with objective measures of anxiety in this cohort, (2) anxiety measures did not demonstrate significant correlation when compared to objective measures for cognitive function, and (3) ABI patients did not display good insight into the severity of their anxiety symptoms as measured by the GAD7. Further research should focus on utilizing other subjective measurement tools for anxiety and/or clinician evaluation tools with NPIQ-A.
Psychological morbidity and associated factors among perinatal patients referred for psychiatry assessments at a tertiary care centre in Sri Lanka

Saumya Madhri Senanayake*, Iresha Perera, Janith Galhenage and Raveen Hanwella

Professorial Psychiatry Unit, National Hospital of Sri Lanka

${ }^{\star}$ Corresponding author.

doi: 10.1192/bjo.2021.766

Aims. Our objective was to study the psychological morbidity and associated risk factors among antenatal and postnatal patients referred for the psychiatric assessment at University Psychiatry Unit of National Hospital of Sri Lanka.

Method. All the Clinic records of perinatal referrals from 1st January 2019 to 31st December 2019 were assessed. Sociodemographic details, delivery details, health of the newborn, past and present psychiatry illness related details were obtained using a questionnaire. Data were analysed using SPSS.

Result. Total of 161 perinatal referrals were studied. Mean age of the mothers were 28.7 years $(\mathrm{SD}=6.60)$. About $18(11.8 \%)$ were not legally married, partner passed away or estranged. Above Ordinary level education was having $34.5 \%$ of participants. Majority were postnatal mothers (61.5\%). Some mothers (32.3\%) have reported the pregnancy was unexpected whilst 20 $(32.3 \%)$ and $49(30.8 \%)$ have experienced delivery complications and neonatal illnesses respectively. Past mental illnesses were found among 31(20.7\%) of mothers. Out of whole perinatal referrals maternity blues $(28.9 \%)$ was the commonest current psychiatry diagnosis. Among antenatal mothers, adjustment disorder (28.8\%) and depressive disorder (17.3\%) were the commonest. Schizophrenia, Schizophreniform disorder and bipolar illness were found among 8(5\%), 6(3.7\%) and 3(1.9\%) mothers respectively. Major psychoactive substance use disorder was found among 4 (2.5\%) mothers. Presence of pregnancy related complications were significantly associated with postpartum metal illnesses $(\mathrm{p}=0.008)$.

Conclusion. Commonest perinatal mental illness was the maternity blues. Depressive disorder was the commonest major mental illness and neonatal complications were associated with psychological morbidity in postnatal mothers.

\section{Attitudes surrounding the disclosure of mental illness}

Brishti Sengupta $^{1 *}$ and Pritha Dasgupta ${ }^{2}$

${ }^{1}$ Dollar Academy and ${ }^{2}$ Stratheden Hospital, NHS Fife ${ }^{*}$ Corresponding author.

doi: 10.1192/bjo.2021.767

Aims. To survey the effect of COVID-19 on mental health of both medical professionals and the general population, as well as attitudes surrounding the disclosure of mental illness.

Method. An online survey comprised of two questionnaires, one for medical professionals and one for the general population, were conducted via social media. Both questionnaires asked respondents of the effect of COVID-19 on their mental health, and the former asked respondents about the effect of COVID-19 on their patient group's mental health. The questionnaires went on to ask respondents about their attitudes to mental health disclosure in various scenarios, to varying groups of people. The general population group was also asked how they would react if someone else disclosed their mental illness to them. 